\title{
Ecto-5'nucleotidase (CD73) May Predict Occurrence and Grade of GVHD in Patients Undergoing Allogeneic Hematopoietic Stem Cell Transplantation
}

Rasha I. Ibrahim*, Maha Elzimaity, Nevine N. Mostafa, Mohamed Hamdy Attia, Walaa A. Elsalakawy, Rana Z. Abass

Hematology and Bone Marrow Transplantation Unit, Department of Internal Medicine, Faculty of Medicine, Ain Shams University, Cairo, Egypt

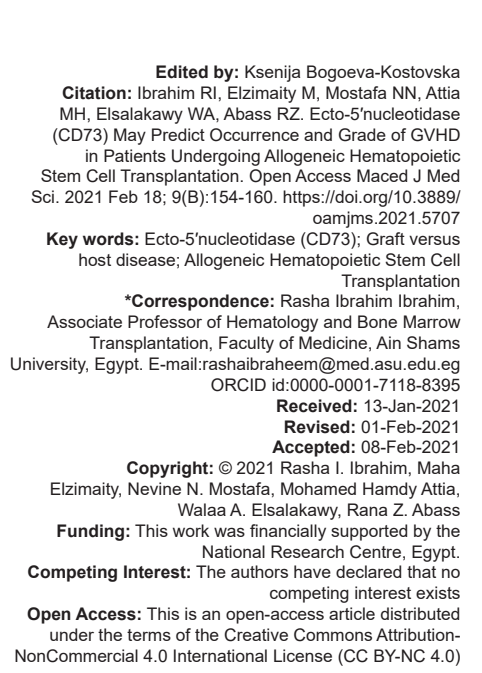

\begin{abstract}
BACKGROUND: Graft versus host disease (GVHD) represents a main cause of post-transplant morbidity and mortality. Ectonucleotidases are one of major components of purinergic signaling which is one of the important mediator pathways regulating cellular functions. CD73 is the most significant member of ectonucleotidases.

AIM: The aim of the study was to assess role of CD73 in development/severity of GVHD among patients undergoing allogeneic hematopoietic stem cell transplantation (HSCT)

SUBJECT AND METHODS: This is a prospective study conducted on 30 adult patients eligible for allogeneic HSCT and their 30 donors in a period of 2 years from January 2015 until January 2017. Assessment of CD73 positive cells through flow cytometry on peripheral blood samples in donors during assessment period before receiving G-CSF and in recipients before conditioning at day -7 and once GVHD occurs (within 12 months) or at end of follow-up period was done.

RESULTS: CD73 level was significantly higher in recipients pre/post-transplantation $(58.24 \pm 19.68,65.78 \pm 19.03$ respectively) than in donors $(29.08 \pm 14.14)(p=0.022)$, there is a significant negative correlation between pretransplant CD73 level in recipients and occurrence of chronic GVHD (cGVHD), $(p=0.004)$ and its grade $(p=0.0496)$ Ectonucleotidase CD73 expression in recipients was a good predictor of cGVHD with sensitivity of $100 \%$ and Specificity of $65 \%$ at cut off value $\leq 61.07 \%$. CD73 expression in recipients was independently predicting cGVHD.

CONCLUSION: CD 73 may represent a promising, clinically applicable tool of predicting CGVHD and its grade in patients undergoing HSCT.
\end{abstract}

\section{Introduction}

Hematopoietic stem cell transplantation (HSCT) is transplantation of multipotent stem cell from bone marrow, peripheral blood (PB) or umbilical cord from the same patient (autologous) or a donor (allogeneic) [1].

Allogeneic hematopoietic cell transplantation is considered as standard curative therapy for several hematological malignancies [2].

Graft versus host disease (GVHD) is the most fatal complication post-allogeneic HSCT and it represents a significant cause of post-transplant morbidity and mortality [3].

GVHD is triggered by alloreactive donor T-lymphocytes with involvement of both CD4+ and CD8+ T cells. The greater the human leukocyte antigen (HLA) mismatches between donors and recipients, the greater the risk of GVHD [4]. Acute GVHD (aGVHD) usually occur within the first 3 months after transplantation with selective affection of skin, liver and intestine. Chronic GVHD (cGVHD) usually starts after 3 months post-transplant and can last a life time with multi organ destruction [5].

aGVHD can occur in up to $50 \%$ of patients receiving HSCT from a HLA-matched sibling [6]. The incidence of cGVHD ranges from $6 \%$ to $80 \%$ [7].

Purinergic signaling modulates inflammation and immune responses on multiple levels and contributes to the pathogenesis of a broad variety of diseases. There are three major components of purinergic signaling: Nucleotides, purinergic receptors, and ectonucleotidases [8].

Ectonucleotidases are nucleotide metabolizing enzymes which possess a major role in regulating the immune system and inflammation. NTPDases (CD39) and ecto-5'nucleotidase (CD73) are the most important members of this group which convert extracellular ATP to ADP, AMP and finally adenosine which bind to and activate adenosine receptors causing either proinflammatory or anti-inflammatory mediator depending on the physiologic setting [9]. 
Thus, CD39/CD73 pathway changes dynamically with the pathophysiological context within which it is embedded. It is becoming progressively recognized that altering this pathway can change the outcome of several pathophysiological events such as AIDS, autoimmune diseases, infections, atherosclerosis, GVHD, and malignancy suggesting their role as novel therapeutic targets for managing a variety of disorders [10].

Different studies have shown that CD73generated adenosine is a factor that can be modified to influence the severity of GVHD [11]. In murine study, it was noticed that GVHD in CD73-/- mice was very severe, increased levels of serum pro-inflammatory cytokines and increased accumulation of $\mathrm{T}$ cells in target tissues which suggest that CD73 generated adenosine limits the severity of GVHD [12].

Most of the studies related to role of CD73 in GVHD were done in murine models so the aim of this work is to assess role of ecto-5'nucleotidase (CD73) in development and severity of acute and cGVHD among Egyptian Adult patients undergoing allogeneic HSCT.

\section{Subjects and Methods}

This is a prospective study which included 30 adult patients eligible for allogeneic HSCT for various hematological diseases and their HLA matched 30 donors (as control) in a period of 2 years from January 2015 until January 2017. Follow-up duration of the patients has been extended up to 12 months posttransplantation to detect clinical outcomes.

All enrolled cases were collected from Bone Marrow Transplantation Unit at Ain Shams University Hospitals; Cairo; Egypt.

All procedures performed in studies were in accordance with the ethical standards of the institutional and/or national research committee and with the 1964 Helsinki declaration and its later amendments or comparable ethical standards. Informed consent was obtained from all individual participants included in the study.

Regarding the diagnosis of patients and conditioning regimens, 13 cases (43.3\%) were diagnosed as acute myeloid leukemia (AML) received fludarabine/busulfan conditioning regimen. Six cases $(20 \%)$ were diagnosed as acute lymphoblastic leukemia (ALL) and received cyclophosphamide/totalbody irradiation regimen conditioning regimen. Six patients $(20 \%)$ were diagnosed as bone marrow aplasia and received antithymocyte globulin/fludarabine/ cyclophosphamide conditioning regimen. Two patients $(6.7 \%)$ were diagnosed as myelodysplastic syndrome and both received fludarabine/busulfan protocol. Another two cases (6.7\%) were diagnosed as non-Hodgkin lymphoma and received busulfan/ cyclophosphamide conditioning regimen. The last case had chronic myeloid leukemia (3.3\%) and received busulfan/cyclophosphamide conditioning regimen.

Bone marrow aspiration and/or biopsy, flowcytometric immunophenotyping and cytogenetic studies were performed at presentation and to confirm remission.

Our patients received stem cells at average dose of $6.29 \pm 1.18 \times 10^{6}$ (with minimum dose was $3.8 \times 10^{6}$ cells $/ \mathrm{kg}$ and maximum dose was $9.1 \times 10^{6}$ cells $/ \mathrm{kg}$ ).

\section{GVHD prophylaxis and grading}

All patients received cyclosporine $A$ (given at a dose of $3-5 \mathrm{mg} / \mathrm{kg} / \mathrm{day}$, it was started at day -1 , given initially intravenously till patient condition permitted oral intake, its dose was adjusted so as to maintain a serum level of $200-400 \mathrm{ng} / \mathrm{ml}$ with twice weekly follow-up which was increased in frequency on demand), and methotrexate at a dose of $10 \mathrm{mg} / \mathrm{kg}$ intravenously at day $+1,+3,+6$, and +11 . This GVHD prophylaxis protocol was maintained for 6-9 months post-transplant. This period shortened when delayed completed donor chimerism took place, or lengthened in case of evolvement of aGVHD.

Early detection of complications particularly GVHD and assessment of its severity were achieved by history, clinical examination, biochemical, histopathological, and radiological investigations according to each disease state.

Staging and grading of aGVHD was done according to the original grading system that was proposed by Glucksberg criteria (Consensus criteria) for staging and grading of aGVHD [13], while diagnosis of cGVHD, classification and severity scoring largely rely on 2005 and 2014 NIH Consensus Conferences [14].

Assessment of CD73 positive cells through flow cytometry on PB samples was done in following occasions:

1. In donors during assessment period before receiving G-CSF

2. In recipients before conditioning (at day -7) and once GVHD occurs (within 12 months) or at end of follow-up period.

- $\quad$ Specimen of enrolled cases' PB was collected in ethylene-diamineteraacetic acid (EDTA)-anti-coagulated blood samples. PB samples were processed on the same day of sample collection

- $\quad$ They were counted using Coulter LH750 cell counter (Beckman Coulter, Hialeah, USA) and the total leukocytic count was adjusted to be around $5.0 \times 10^{9} / \mathrm{L}$ using phosphate buffered saline $120 \mathrm{mM} \mathrm{NaCl}, 2.7 \mathrm{mM} \mathrm{KCl}, 10$ 
gastrointestinal GVHD, three of them (50\%) had isolated gut aGVHD, one patient had combined acute hepatic and gut GVHD, one patient had mucocutaneous and gut GVHD, and another patient had combined skin, hepatic, and gut aGVHD. The other 24 patients (80\%) did not expertise aGVHD, but 8 of them (26.7\%) died within first 2 months.

\section{cGVHD}

Concerning CGVHD, its incidence was higher in our study than aGVHD as ten patients $(33.3 \%)$ were affected. Six of them (60\%) had a moderate grade, two patients $(20 \%)$ had a mild grade, while the last two cases (20\%) had severe grade. According to specific organ affection in cGVHD, six patients $(20 \%)$ had chronic hepatic GVHD 2 of them were progressive overlap and other 4 were classic de novo, four patients (13.3\%) had mucocutaneous cGVHD, 2 of them were overlap progressive and other 2 classic de novo, 3 cases $(10 \%)$ had chronic pulmonary GVHD classic de novo type, while two patients $(6.7 \%)$ had chronic classic de novo gut GVHD. The remaining 20 patients $(66.7 \%)$ did not have any manifestations of cGVHD, however, 13 patients of them $(43.3 \%)$ died before having cGVHD.

Ecto-5'nucleotidase (CD73) level assessment and correlations (in donors and recipients pre- and post-transplant):

Average value of pre-transplant CD73 level (\%) in recipients was $58.24 \pm 19.68$, while its level posttransplant (at time of GVHD or at the end of follow-up) was of $65.78 \pm 19.03$, while in donors CD73 mean value was $29.08 \pm 14.14$ (Table 1).

There is a highly significant statistical difference between patients developed cGVHD and those who did not, as regards pre-transplant CD73 level (Table 3$)$, with a higher mean value $(65.2 \pm 17.8 \%)$ in patients group who did not develop cGVHD compared to patients group who had cGVHD (mean value $44.4 \pm$ 16.2); $(p=0.004)$. Thus, lower values of pre-transplant CD 73 in recipients were associated with occurrence of cGVHD.

Table 3: Relationship between the pre-transplantation CD73 expression in recipient or donors and the occurrence of GVHD

\begin{tabular}{|c|c|c|c|c|c|c|c|c|}
\hline \multirow[t]{3}{*}{ Outcome } & \multicolumn{8}{|c|}{ Pre-transplantation CD73 expression (\%) } \\
\hline & \multicolumn{4}{|c|}{ Recipients } & \multicolumn{4}{|c|}{ Donors } \\
\hline & $\mathrm{n}$ & Mean & SD & $p$-value* & $\mathrm{n}$ & Mean & SD & p-value \\
\hline \multicolumn{9}{|l|}{ Acute GVHD } \\
\hline No acute GVHD & 24 & 60.2 & 18.2 & $0.270(\mathrm{NS})$ & 24 & 29.4 & 15.6 & 0.795 (NS) \\
\hline Acute GVHD & 6 & 50.2 & 25.2 & & 6 & 27.7 & 5.9 & \\
\hline \multicolumn{9}{|l|}{ Chronic GVHD } \\
\hline No chronic GVHD & 20 & 65.2 & 17.8 & 0.004 (HS) & 20 & 31.0 & 16.7 & 0.307 (NS) \\
\hline Chronic GVHD & 10 & 44.4 & 16.2 & & 10 & 25.3 & 5.9 & \\
\hline
\end{tabular}

We noticed a significant negative correlation between recipient's pre-transplant $C D 73$ expression and grade of cGVHD (Spearman rho $=0.812, p=0.0499$ ), but there is no correlation between aGVHD and CD73 levels pretransplant and in donors (Tables 3 and 4).
Table 4: Correlation between CD73 expression in donors and recipients (pre/post-transplant) with grading of acute and chronic GVHD

\begin{tabular}{|c|c|c|c|c|}
\hline & Grade of acute & VHD & Grade of chron & GVHD \\
\hline Variable & Spearman rho & $p$-value & Spearman rho & $p$-value \\
\hline $\begin{array}{l}\text { Recipient's CD73 } \\
\text { before transplantation }\end{array}$ & -0.245 & 0.496 (NS) & -0.812 & $0.0499(\mathrm{~S})$ \\
\hline Donor's CD73 & 0.493 & 0.321 (NS) & 0.289 & 0.417 (NS) \\
\hline $\begin{array}{l}\text { Recipient's CD73 } \\
\text { after transplantation }\end{array}$ & -0.232 & 0.658 (NS) & 0.491 & 0.150 (NS) \\
\hline
\end{tabular}

expression $\leq 27.5 \%$ could predict aGVHD with a sensitivity of $33.3 \%$ and specificity of $100 \%$. While Donors' CD73 expression $>30 \%$ could predict aGVHD with a sensitivity of $33.3 \%$ and specificity of $87.5 \%$. Regarding cGVHD, pre-transplant CD73expression in recipients had good predictive value $(A U C=0.843)$ with sensitivity of $100 \%$ and specificity of $65 \%$ at cut off value $\leq 61.07 \%$. While in donors, it had poor predictive value (AUC $=0.663$ ) with a sensitivity of $60 \%$ and specificity of $80 \%$ at levels $\leq 27.08 \%$ (Figure 1 ).

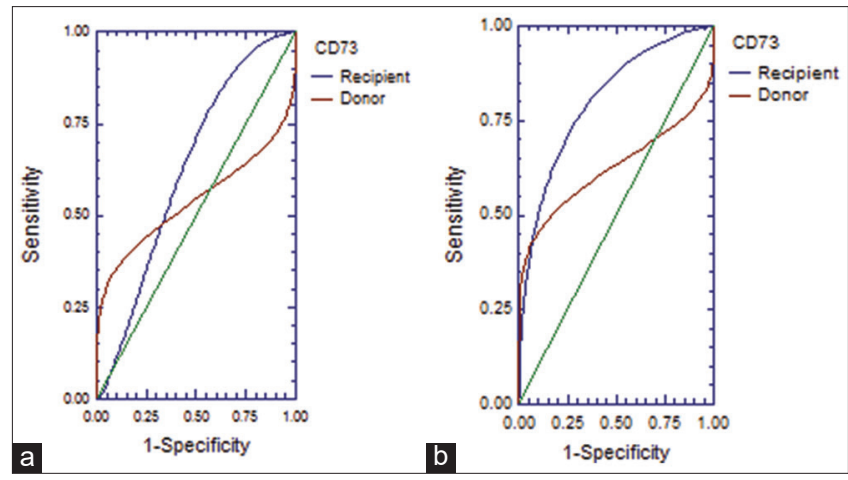

Figure 1: (a) Receiver-operating characteristic (ROC) curves for prediction of acute graft versus host disease (GVHD) using pre-transplantation CD73 expression in the recipient or in the donor. (b) ROC curves for prediction of chronic GVHD using pre-transplantation $C D 73$ expression in recipients or donors

Competing risks regression analysis for cGVHD showed that CD73 expression in recipients wasindependently predictingcGVHD but not aGVHD (Table 5). While neither the recipients' CD73 expression pre-transplant $(\mathrm{SHR}=0.980,95 \% \mathrm{Cl}=0.942-$ 1.021, $p=0.340$ ) nor the donors' (SHR $=1.005,95 \%$ $\mathrm{Cl}=0.963-1.048, \mathrm{p}=0.832)$ was an independent predictor for acute GVHD.

\section{Discussion}

Bone marrow transplant is one of the few curative modalities in the field of hematological diseases. Despite recent advances, GVHD is still a significant cause of post-transplant morbidity and mortality. Drugs used to prevent or treat GVHD are toxic and they themselves can cause further morbidities and mortalities. Thus, clinicians are in need to new tools to predict GVHD to modify the dose and regimen of anti-GVHD protocols. 
Table 5: Competing risks regression analysis for cGVHD

\begin{tabular}{|c|c|c|c|c|c|c|}
\hline \multirow[t]{2}{*}{ Variable } & \multirow[t]{2}{*}{ Robust SHR } & \multirow[t]{2}{*}{ SE } & \multirow[t]{2}{*}{$\mathrm{Z}$} & \multirow[t]{2}{*}{$\mathrm{p}$-value } & \multicolumn{2}{|c|}{$95 \% \mathrm{Cl}$} \\
\hline & & & & & Lower & Upper \\
\hline Recipient's CD73 expression before transplantation (\%) & 0.963 & 0.011 & -3.190 & 0.001 & 0.940 & 0.985 \\
\hline Donor's CD73 expression (\%) & 0.997 & 0.024 & -0.130 & 0.895 & 0.951 & 1.045 \\
\hline
\end{tabular}

Ectonucleotidases are family group of nucleotide metabolizing enzymes which possess a major role in regulating the immune system and inflammation.

In the current study, we investigated if the level of CD73 expression may predict the occurrence or the grade of GVHD whether acute or chronic. The study was conducted on 30 adult patients eligible for allogeneic HSCT for different hematological diseases and their 30 donors.

We found that CD73 level (\%) was higher in recipients pre-and post-transplantation $(58.24 \pm 19.68$, $65.78 \pm 19.03$, respectively) than in donors $(29.08 \pm$ 14.14); $(p=0.022)$.

This finding may be explained as $43.3 \%$ of our patients had AML, and another $20 \%$ diagnosed as ALL which was in agreement with Bastid et al. who showed that CD73 has high expression level and activity in several blood and solid tumors suggesting its role in promoting tumor growth and infiltration [15].

Furthermore, Zhao et al. had investigated CD73 expression in various leukemia subtypes and revealed that $\mathrm{CD} 73$ expression was related to leukemia subtype with high expression in acute lymphocytic leukemia type $B$ and other subtypes of $A M L$ specially (M1, M2a, t (8; 21), t (15; 17), M4 and M5) compared with healthy individuals [16].

Another clarification of this finding was processed by Samanta et al. who found that chemotherapeutic agents can induce expression of CD73 as well as hypoxia induced factor 1 (HIF-1 $\alpha$ ), and (HIF-2 $\alpha$ ) that successively induces more expression of CD73 [17].

In our study, aGVHD was present in $20 \%$ of patients (six recipients), while Jacobsohn and Vogelsang stated that aGVHD remains a major complication of allogeneic transplantation occurring in approximately half the transplanted recipients [18].

Another study showed that the incidence of aGVHD about $9-50 \%$ in HLA matched donors and up to $75 \%$ in unrelated matched donors [19]. All our patients had fully HLA matched related donor.

Review by Villarreal et al., 2016 revealed aGVHD as major cause of morbidity and mortality affecting $40-60 \%$ of recipients after allogeneic HSCT with mortality rate about $15 \%$ [20].

Incidence of gastrointestinal aGVHD in our study was $20 \%$, cutaneous was $6.7 \%$, and hepatic aGVHD was $6.7 \%$. While Cutler and Antin showed that cutaneous involvement is the most common type of aGVHD [21].

Our current results showed that there was no significant statistical differences regarding pretransplantation CD73 levels between patients' group who had aGVHD (50.2 \pm 25.2$)$ compared to the other group who did not have aGVHD $(60.2 \pm 18.2)$, however, its level was higher in patients without aGVHD, thus we may need large groups of patients to prove its statistical significance.

Jones and Kang discussed the protective role of CD73 in GVHD development. They showed that CD73 generated adenosine produce immunosuppressive effect by activating A2A receptor pathway which inhibits the activation of effector T cells, release of proinflammatory cytokines such as interleukin-1 (IL-1), IL-6, IL-12, and tumor necrosis factor- $\alpha$ and increases the overall number of T-regs which have proven to be effective in reducing and preventing GVHD development in murine models [4].

This result was also compatible with findings of murine study done by Wang et al. on mice which showed that CD73 plays a critical role in the T cellmediated development of aGVHD. They proved that CD73 KO donor T cells have the ability to proliferate and infiltrate host tissues leading to cytokine storm with release of multiple pro-inflammatory cytokines causing systemic aGVHD [22].

The same results were reported in murine study which used gene-targeted mice and a pharmacologic inhibitor of CD73 and revealed that CD73 deficiency was associated with increased incidence and severity of acute GVHD [11].

Tsukamoto et al. showed that both donor and recipient CD73 were relevant for tolerogenic effects and deficiency in either one of them caused enhanced GVHD; however, recipient CD73 played a more prominent role [11]. The same finding was reported by Wang et al. who also proved that donor CD73 KO regulatory $T$ cells (Treg) had significantly impaired the ability to mitigate GVHD mortality compared to WT Treg; however, recipient CD73 is more effective in limiting GVHD [22]. On the other hand, in our study there was no statistical significance of CD73 level in donors with the incidence of either acute or cGVHD in recipients ( $p=0.795,0.307$, respectively) which may be explained by differences in human and murine models.

Results of the current study showed no significant correlation between pre-transplant CD73 level in recipients and aGVHD grading and CD73 
expression in recipients was not a good predictor of aGVHD with a sensitivity of $33.3 \%$.

To our knowledge, no similar studies were done on humans before but Thompson et al. study revealed that the severity of aGVHD is significantly higher when mismatched bone marrow transplants are performed between Cd73-/- mice with severe histopathological finding in affected organs [12].

Wang et al. also reported similar results after examining pathological finding in lung, liver, skin, and colon in CD73-/- recipients mice compared with wild type and showed increased severity and scoring of aGVHD between CD73-/- recipients [22].

Furthermore, in our study, there was no correlation between CD73 in donors with aGVHD grading ( $p=0.289)$. This is the contrast to Wang et al. study which showed that deficiency of CD73 in both recipients and donors play a significant role in GVHD development and severity [22]. The same results were reported by Tsukamoto et al. who also proved the role of donors' CD73 on severity of GVHD [11]. This may need more research on human to clarify its significance.

As regard cGVHD, it occurred in $33.3 \%$ of our patients $(n=10)$ and this is in agreement of Lee and Flowers results who documented that cGVHD was the most serious and common long-term complication of allo-HSCT, occurring in 30-70\% of adults and children surviving more than 100 days [23].

CD73 was significantly higher in patients who did not develop cGVHD in comparison to those who developed cGVHD (65.2 \pm 17.8 versus $44.4 \pm$ 16.2 respectively, $p=0.004)$, and there was negative correlation between pre-transplantation CD73 expression in recipients with severity of cGVHD. Our results additionally showed that CD73 expression in recipients was a good predictor of cGVHD with sensitivity of $100 \%$ and specify of $65 \%$ at cutoff value $\leq 61.07$. On doing multivariate competing risk regression analysis of predictors of CD73 expression in recipients, we found that CD73 expression in recipients was independently predicting $C G V H D$.

Immune system has a critical role in cGVHD pathogenesis, as cGVHD reflects a state of inability to achieve immune tolerance resulting in persistence of allo and autoreactive $\mathrm{T}$ and $\mathrm{B}$ cells. Donor alloreactive $T$ cells recognize the host target tissues leading to their damage by cytokine release and direct cytolysis. In the same time, mature donor $\mathrm{T}$ cells within the graft contribute to thymic destruction resulting in disrupted immune reconstitution. There is also reduction in T-reg cells during cGVHD which is associated with loss of immune tolerance and development of autoimmunity. Altered B-regs and NK development after SCT is thought to contribute to cGVHD as patients with cGVHD had reduced levels of circulating IL-10-producing B-regs and impaired
IL-10 production [24].

Our study was limited by small number of patients and it is considered novel in human being, we need more large studies on human to clarify exact role of ectonucleotidases especially CD73 in HSCT and its complications.

\section{Conclusions}

Better understanding of the possible underlying mechanisms responsible for GVHD and the involved molecules will lead to better prevention and treatment regimens. Markers that could predict GVHD is a real clinical challenge. CD 73 is a promising clinical tool as CD 73 may predict occurrence and severity of cGVHD in patients undergoing HSCT.

\section{Statement of ethics}

The case was approved by the ethical committee of our center and the patient signed a written informed consent to publish the case (including publication of images).

\section{Availability of data and materials}

The data sets during and/or analyzed during the current study available from the corresponding author on reasonable request.

\section{Authors' Contributions}

All authors equally contributed in witting and editing

\section{References}

1. Robbie N, Deborah T. Hematopoietic stem cell transplantation In: Tomlinson D, Kline NE, editors. Pediatric Oncology Nursing. Advanced Clinical Hand Book. $2^{\text {nd }}$ ed., Ch. 3. Germany: Springer; 2010. p. 243-67.

2. Apostolova P, Zeiser R. The Role of purine metabolites as DAMPs in acute graft-versus-host disease. Front Immunol. 2016;7:439. https://doi.org/10.3389/fimmu.2016.00439 PMid:27818661

3. Martin B, Deeg H. acute graft versus host disease. In: Soiffer RJ, editor. Hematopoietic Stem Cell Transplantation. $2^{\text {nd }}$ ed., Vol. 1, Ch. 25. United States: Humana Press; 2008. p. 589-620. https:// doi.org/10.1007/978-1-59745-438-4_25 
4. Jones KR, Kang EM. Graft versus host disease: New insights into A2A receptor agonist therapy. Comput Struct Biotechnol J. 2014;13:101-5.

PMid:25709759

5. Marks C, Stadler M, Häusermann $P$, Wolff D, Buchholz $S$ Stary G, et al. German-Austrian-Swiss consensus conference on clinical practice in chronic graft-versus-host disease (GVHD): Guidance for supportive therapy of chronic cutaneous and musculoskeletal GVHD. Br J Dermatol. 2011;165(1):18-29. https://doi.org/10.1111/j.1365-2133.2011.10360.x PMid:21466534.

6. Ferrara JL, Levine JE, Reddy P, Holler E. Graft-versushost disease. Lancet. 2009;373(9674):1550-61. https://doi. org/10.1016/s0140-6736(09)60237-3

PMid:19282026

7. Atkinson K, Horowitz MM, Gale RP, van Bekkum DW, Gluckman E, Good RA, et al. Risk factors for chronic graftversus-host disease after HLA-identical sibling bone marrow transplantation. Blood. 1990;75(12):2459-64. https://doi. org/10.1182/blood.v75.12.2459.bloodjournal75122459 PMid:2350582

8. Robson SC, Sévigny J, Zimmermann H. The E-NTPDase family of ectonucleotidases: Structure function relationships and pathophysiological significance. Purinergic Signal. 2006;2(2):409-30. https://doi.org/10.1007/s11302-006-9003-5 PMid: 18404480

9. Antonioli L, Pacher P, Vizi ES, Haskó G. CD39 and CD73 in immunity and inflammation. Trends Mol Med. 2013;19(6):35567. https://doi.org/10.1016/j.molmed.2013.03.005 PMid:23601906

10. Zhang B. CD73 promotes tumor growth and metastasis. Oncoimmunology. 2012;1(1):67-70. https://doi.org/10.4161/ onci.1.1.18068

PMid:22720214

11. Tsukamoto H, Chernogorova $\mathrm{P}$, Ayata $\mathrm{K}$, Gerlach UV, Rughani A, Ritchey JW, et al. Deficiency of CD73/ecto5'-nucleotidase in mice enhances acute graft-versus-host disease. Blood. 2012;119(19):4554-64. https://doi.org/10.1182/ blood-2011-09-375899

PMid:22262774

12. Thompson LF, Tsukamoto $H$, Chernogorova P, Zeiser R. A delicate balance: $C D 73-g e n e r a t e d$ adenosine limits the severity of graft vs. host disease but also constrains the allogeneic graft vs. tumor effect. Oncoimmunology. 2013;2(1):e22107. https:// doi.org/10.4161/onci.22107

PMid:23482243

13. Przepiorka D, Weisdorf D, Martin P, Klingemann HG, Beatty $P$, Hows J, et al. 1994 consensus conference on Acute GVHD grading. Bone Marrow Transplant. 1995;15(6):825-8. PMid:7581076

14. Carpenter PA, Kitko CL, Elad S, Flowers ME, GeaBanacloche JC, Halter JP, et al. National institutes of health consensus development project on criteria for clinical trials in chronic graft-versus-host disease: V. The 2014 ancillary therapy and supportive care working group report. Biol Blood Marrow Transplant. 2015;21(7):1167-87. https://doi.org/10.1016/j. bbmt.2015.03.024

PMid:25838185

15. Bastid J, Cottalorda-Regairaz A, Alberici G, Bonnefoy N, Eliaou JF, Bensussan A. ENTPD1/CD39 is a promising therapeutic target in oncology. Oncogene. 2013;32(14):174351. https://doi.org/10.1038/onc.2012.269 PMid:22751118

16. Zhao SX, Zhang HM, Dong SX, Liu JH, Zhou Z, Wang HJ, et al Characteristics and clinical significance of CD73 expression in subtypes of leukemia. Zhongguo Shi Yan Xue Ye Xue Za Zhi. 2011;19(5):1141-4

PMid:22040959

17. Samanta D, Park Y, Ni X, Li H, Zahnow CA, Gabrielson E, et al. Chemotherapy induces enrichment of CD47+/CD73+/PDL1+ immune evasive triple-negative breast cancer cells. Proc Nat Acad Sci USA. 2018;115(6):E1239-48. https://doi.org/10.1073/ pnas. 1718197115 PMid:29367423

18. Jacobsohn DA, Vogelsang GB. Acute graft versus host disease. Orphanet J Rare Dis. 2007;2:35. https://doi. org/10.1186/1750-1172-2-35

PMid: 17784964

19. Krstevska S, Genadieva-Stavric S, Pivkova A, Stojanovski Z, Georgievski B, Balkanov T. Acute graft versus host disease in hematopoietic stem cell alotransplant recipients. Med Arh. 2011;65(5):260-4. https://doi.org/10.5455/ medarh.2011.65.260-264

PMid:22073847

20. Villarreal CD, Alanis JC, Pérez JC, Candiani JO. Cutaneous graft-versus-host disease after hematopoietic stem cell transplant-a review. An Bras Dermatol. 2016;91(3):336-43. https://doi.org/10.1590/abd1806-4841.20164180 PMid:27438202

21. Cutler $\mathrm{C}$, Antin JH. Manifestations and treatment of acute graftversus-host disease. In: Frederick RA, Stephen JF, Robert SN, Karl GB, editors. Thomas' Hematopoietic Cell Transplantation. $4^{\text {th }}$ ed., Ch. 86. United States: Wiley Blackwell; 2009. p. $1287-$ 303. https://doi.org/10.1002/9781444303537.ch86

22. Wang L, Fan J, Chen S, Zhang Y, Curiel TJ, Zhang B. Graftversus-host disease is enhanced by selective CD73 blockade in mice. PLoS One. 2013;8(3):e58397. https://doi.org/10.1371/ journal.pone.0058397 PMid:23520507

23. Lee SJ, Flowers ME. Recognizing and managing chronic graft-versus-host disease. Hematology Am Soc Hematol Educ Program. 2008:134-41. https://doi.org/10.1182/ asheducation-2008.1.134 PMid:19074071

24. Min CK. The pathophysiology of chronic graft-versus-host disease: The unveiling of an enigma. Korean $\mathrm{J}$ Hematol. 2011;46(2):80-7. https://doi.org/10.5045/kjh.2011.46.2.80 PMid:21747879 